\title{
CORRESPONDENCE
}

\section{A PRIZE FOR SYMBIOSIS}

\author{
Ingo Althøfer \\ Fakultăt für Mathematik \\ Universităt Bielefeld \\ Postfach 8640 \\ 4800 Bielefeld 1 \\ Federal Republic of Germany
}

Probably it will still take many years before a chess computer will be able to dethrone the human chess world champion. One of the main reasons for this is, in my estimation, that none of the major present-day chess computers is able to realize long-term strategic plans. This shortcoming could be alleviated by combining machine tactics with human strategic insights.

I propose a symbiosis: System $S$ (the Symbiotic System) consists of two parts, namely a chess computer and one human player. If System $S$ has to make a move in a chess game, the computer first produces a list of at least one and at most three moves. (The number three is my choice and might be replaced by any other limit) This list is given to the human partner, who selects the definitive move from it and executes this move on the board. This decision sharing achieves two objectives:

- The danger of a positional or strategic error made by the computer is reduced.

- The danger of a tactical blunder made by the human player is reduced.

In order to promote the testing of this idea, I offer a prize of 10,000 DM for the first combination of a chess computer and a human player winning at least 3 points in a 6-game match against the current chess world champion.

The precise conditions are as follows:

My offer is limited in time. The match must begin before December 31, 1995 and it must end before January 31,1996 . The match is to be under the FIDE rules holding for the 1986 Kasparov-Karpov contest (each player has 2.5 hours for the first 40 moves, after 5 hours the game is adjourned and not resumed before the next day, and so on). System $\mathrm{S}$ has to execute four tasks when its clock is running: entry of the actual position into the computer; production of the move list by the computer; selection of the definitive move by the human part; execution of this move on the board. The list of proposed moves, produced by the computer whenever System $S$ is to move, must contain at least one and at most three moves. The computer should clearly signal the end of the move list. The special case in which the computer always proposes exactly one move is allowed. (In this case the world champion would be playing directly the computer of System S.) Several chess computers may be combined as a network. Of course, it has to be established that no human can interfere with the computer. A referee has to check that the computer proposes no more than three moves and that the human partner of System $S$ selects a move only from among the ones proposed. The prize goes to the first contender finishing his 6-game match with a score of 3 points or better. The world champion is excluded as the human partner of System S. The human partner is allowed to override the computer suggestions at most three times during a game, which decision may only be made after inspection of the moves proposed. 